\title{
2016-17 List of Reviewers / \\ Les examinateurs de la RHÉ pour l'année 2016-2017
}

Katrina Ackerman
Funke Aladejebi
Michael Allard
Tim Allender
John Allison
Linda Ambrose
Marie Averginou
Paul Axelrod
Jean Barman
Joel Belliveau
Louise Bienvenue
Pablo Blanco
Felix Bouvier
Catherine Broom
Lorne Bruce
William Bruneau
Kevin Brushett
Rosa Bruno-Jofré
Sara Burke
Timothy Cain
Mathilde Cambron-
Goulet
Sean Carleton
Adam Chapnick
Mary Chaktsiris
Jessica Clark
Cynthia Comacchio
Rebecca Coulter
Bruce Curtis
Bambi Ceuppens
Eric Damer
Kari Dehli

Anthony Di Mascio
Jack Dougherty
G. Antonio Espinoza
Susan Fisher
Thomas Fleming
Catherine Foisy
Catherine Gidney
Jennifer Gilbert
Dalie Giroux
Alastair Glegg
Christopher Greig
Christine Hudson
Alyson King
Madeline Knickerbocker
Adam Laats
Kathryn Labelle
Laurier Lacroix
Guy Laperrière
Lynn Lemisko
Charles Levi
Roberta Lexier
Drew Lopenzina
Brittany Luby
Heather E. McGregor
Peter F. McNally
Michael Marker
Alison Marshall
Dominique Marshall
Marcel Martel
Jane Martin
Patrice Milewski

W. P. J. Millar

J. R. Miller

Sean Mills

Jocelyne Murray

Tamara Myers

Nicole Neatby

Susan Neylan

Kenneth Osborne

Doug Owram

Kate Pahl

Adele Perry

Helen Raptis

Kathryn Ricketts

Kate Rousmaniere

Amy Samson

Ruth Sandwell

Harry Smaller

Meaghan Scanlon

Alan Sears

Marlene Shore

Kael Sharman

Harry Smaller

Elizabeth Smyth

Ruth-Ellen St. Onge

Gerald Thomson

Brian Titley

Jennifer Tupper

Karen Vallgårda

Patricia Vertinsky

Martha Walls

Jean-Philippe Warren 
170 Historical Studies in Education/Revue d'histoire de l'éducation 